\title{
DEPRESIÓN Y ESTILOS DE AFRONTAMIENTO AL ESTRÉS EN PACIENTES CON ENFERMEDAD CEREBROVASCULAR
}

\author{
DEPRESSION AND COPING STYLES OF STRESS IN PATIENTS WITH STROKE
}

\author{
Dennys BarReda S $^{1}$ \\ Universidad Nacional Mayor de San Marcos, Perú \\ (RECiBIDo (02/07/2012 - AcEPTADo 30/11/2012)
}

\begin{abstract}
RESUMEN
La enfermedad cerebrovascular (ECV) se define como la interrupción súbita del flujo sanguíneo de los vasos en cualquier área del cerebro. La presencia de depresión se da con suma regularidad en las personas afectadas. Asimismo, las personas que padecen mayor estrés son candidatos potenciales a padecer una ECV. Por tal motivo, la presente investigación tiene por objetivo describir la relación entre la depresión y los estilos de afrontamiento al estrés en una muestra de 50 pacientes con enfermedad cerebrovascular, atendidos en consulta externa en el servicio de neurología del hospital Daniel Alcides Carrión. Utilizándose para ello el Inventario de Depresión de Beck y el Inventario de Estimación de Afrontamiento en su forma disposicional breve de Carver. Se encontró una correlación estadísticamente significativa entre las variables depresión y estilos de afrontamiento al estrés evitativo, sin embargo, no se hallaron correlaciones significativas entre las variables depresión y estilos de afrontamiento al estrés centrados en el problema y centrados en la emoción respectivamente.
\end{abstract}

Palabras clave: Enfermedad cerebrovascular, depresión, estilos de afrontamiento, estrés

\begin{abstract}
The stroke is defined as the sudden interruption of blood flow in vessels in any area of the brain. The presence of depression is given with great regularity in those affected. Also, people with more stress are potential candidates for developing stroke. For this reason, this research describe the relationship between depression and stress coping styles, in a sample of 50 stroke patients treated at the outpatient service of neurology at Daniel Alcides Carrion Hospital . For this purpose used the Beck Depression Inventory and Brief COPE-The Coping Estimation Inventory. This research found a statistically significant correlation between the depression's variables and coping styles to stress avoidance, however there were no significant correlations between the depression's variables and stress coping styles focused on the problem and emotion respectively.
\end{abstract}

Keywords: depression, coping style, stroke, stress. 


\section{INTRODUCCIÓN}

En el Perú, la enfermedad cerebrovascular (ECV) constituye la principal causa de discapacidad en personas mayores de 45 años, (Velásquez, 2006). Esta enfermedad es una patología importante en el hospital Nacional Daniel Alcides Carrión (HNDAC) y una causa frecuente de incapacidad para la persona que lo sufre.

En el área funcional de consulta externa del servicio de neurología de la referida institución, el grupo de las enfermedades cerebrovasculares codificada entre los rangos (I60-I69) según la Organización Mundial de la Salud (1992), es la principal causa de atención (Área de Procesamiento de Datos del HNDAC, 2009).

La enfermedad cerebrovascular se define como la interrupción en el flujo sanguíneo de los vasos, en cualquier área del cerebro, pudiendo afectar los sentidos, de un momento a otro, el habla, el procesamiento de ideas, la memoria, el comportamiento, o provocar trastornos motores, estado de coma o la muerte; constituyendo una prioridad sanitaria debido a su impacto y las repercusiones que se dan en la calidad de vida de las personas afectadas con este mal (Arrazola, Beguiristain, Garitano, Mar y Elizalde, 2005).

La aparición de depresión posterior a una ECV es un factor importante, debido a que se da con suma regularidad en estos pacientes. En los últimos años, ésta ha sido ampliamente estudiada y reconocida como una entidad particular que afecta a los pacientes que presentan secuelas neurológicas a consecuencia de la enfermedad cerebrovascular (Rojas, 2002).

Este alcance es compatible con el que se indica en la Décima Clasificación Internacional de Enfermedades (CIE 10), la cual define a los episodios depresivos como aquellos en los que la persona sufre un humor depresivo, pérdida de la capacidad de interesarse y disfrutar las cosas, una disminución de su vitalidad que lleva una reducción de su nivel de actividad y a un cansancio exagerado, que aparece incluso tras un esfuerzo mínimo, también se presentan manifestaciones como, pérdida de confianza en sí mismo y sentimientos de inferioridad, las ideas de culpa y de ser inútil, así como una perspectiva sombría del futuro, entre otras (Organización Mundial de la Salud, 1992).

El modelo cognitivo postula que para comprender el cuadro depresivo es necesario tener presente tres conceptos esenciales, estos son la tríada cognitiva, los esquemas y los errores cognitivos; la tríada cognitiva se caracteriza porque la persona tiene una visión negativa de sí mismo, de sus experiencias en el mundo y el futuro; esta visión negativa está ligada a los esquemas los cuales son los patrones cognitivos que regirán la manera de estructurar las experiencias, estos a su vez mantienen los errores cognitivos, por los que el individuo depresivo sostiene la creencia de validez de sus interpretaciones negativas a pesar de evidencias contrarias (Beck, 1983).

Asociado también a la ECV, se encuentran los estilos de afrontamiento que usualmente utiliza la persona para manejar las situaciones difíciles de la vida diaria, así pues, como mencionan Surtees, Wainwright, Luben, Wareham, Bingham \& 
Khaw (2008), las personas que padecen mayor estrés psicológico son candidatos potenciales para sufrir un accidente cerebrovascular (ACV) y aquellos que son capaces de adaptarse más rápidamente a las circunstancias estresantes de sus vidas tienen un riesgo menor de ACV (Surtees, Wainwright, Luben, Wareham, Bingham \& Khaw, 2007).

Para Lazarus y Folkman (1986, p. 43), “el estrés psicológico es una relación particular entre el individuo y el entorno que es evaluado por éste como amenazante o desbordante de sus recursos y que pone en peligro su bienestar". Los autores mencionados consideran esta situación a través del análisis de los dos procesos críticos que tienen lugar en relación al individuo-entorno, la evaluación cognitiva y el afrontamiento; la evaluación cognitiva es un proceso evaluativo que determina por qué y hasta qué punto, una relación determinada o una serie de relaciones entre el individuo y el entorno es estresante y el afrontamiento es el proceso a través del cual el individuo maneja las demandas de la relación individuo-ambiente que evalúa como estresantes y las acciones que ello genera. Cuando la persona utiliza con regularidad ante situaciones estresantes, respuestas características se puede hablar del uso de estilos de afrontamiento. Los tres estilos de afrontamiento generales son los siguientes (Lazarus y Folkman, 1986; Carver, Scheier \& Weintraub, 1989).:

a) El afrontamiento centrado en el problema consiste en la utilización de recursos dirigidos hacia sí mismo o hacia el ambiente, obteniendo información acerca de la mejor manera de modificar la situación problemática, para luego realizar las acciones pertinentes. Comprende las siguientes estrategias de afronte dirigidos a modificar el estresor:

»Afrontamiento activo, se define como aquel proceso por el cual la persona ejecuta acciones directas, aumentando los esfuerzos necesarios destinados a evitar, apartar o disminuir los efectos negativos.

» Planificación, implica el planeamiento mental que realiza el individuo, pensando en la manera de manejarse mediante la organización de estrategias de acción, los pasos a seguir y el establecimiento de la mejor forma de manejar el problema o estresor.

» Búsqueda de apoyo social por razones instrumentales, es decir, buscar en los demás recursos materiales, tales como consejo, asistencia e información, con la finalidad de dar solución al problema.

b) El afrontamiento centrado en la emoción tiene por objetivo regular las emociones que son activadas por el hecho percibido como amenazante, a partir de evaluaciones cognitivas que permiten revalorizar la amenaza modificando así las emociones y con ello, el significado de la relación estresante. Comprende las siguientes estrategias:

» Búsqueda de apoyo social por razones emocionales, implica buscar, ya sea la comprensión de los demás, apoyo moral y empatía con el objetivo de mitigar la reacción emocional negativa.

»Reinterpretación positiva y crecimiento, indica que en diversas ocasiones la persona evalúa el problema atribuyendo una relación positiva a la experiencia en sí misma o bien tomándola como un aprendizaje para el futuro. 
» La aceptación, comprende dos aspectos durante el proceso de afrontamiento. El primero se realiza en la fase de evaluación primaria, cuando la persona admite la existencia de la situación estresante; el segundo aspecto se da durante la evaluación secundaria en donde, debido a que no se puede modificar tal estresor, se acepta convivir con él.

» Acudir a la religión, se refiere a la tendencia a volcarse en la religión con la intención de mitigar la tensión existente en la situación problemática.

» La negación, consiste en resistirse a creer que el estresor existe o tratar de pensar y actuar como si éste no fuera real.

» Humor, consiste en considerar la situación restándole importancia o aliviando su efecto crítico mediante el uso de bromas.

c) El estilo de afrontamiento evitativo se presenta cuando la persona se desentiende del manejo del estresor mental y conductualmente, ya que este interfiere en el logro de su objetivo, se muestra una conducta caracterizada por un insuficiente esfuerzo para controlar el conflicto y en el aspecto mental, la persona tiende a dispersar sus pensamientos, realizando una serie de actividades que no conllevan al manejo de la dificultad. La persona tenderá a desentenderse del estresor mediante los siguientes estilos de afronte específicos:

»Enfocar y liberar emociones, consiste en centrarse en las consecuencias emocionales desagradables experimentadas, expresándolas abiertamente.

»Desentendimiento conductual, implica aminorar los esfuerzos dirigidos hacia el estresor, abandonando las metas que son obstruidas por la situación crítica.

» Autodistracción, consiste en permitir una amplia variedad de actividades que distraigan a la persona de la evaluación de las dimensiones conductuales del problema o de la meta obstruida por el estresor.

» Uso de sustancias, implica el consumo de alcohol u otras drogas con la finalidad de evadir la situación estresante.

»Autoculpa, esta escala nueva se refiere a la autoimputación, sentimiento de responsabilidad o por un castigo a consecuencia de alguna acción u omisión realizada.

Debido a la alta complejidad del ser humano en donde entran en juego una serie de variables biológicas, ecológicas, sociales, espirituales y psicológicas, en su constitución y por ende en su estado de salud; en la enfermedad cerebrovascular juegan un rol importante diversos factores que incidirán en la calidad de vida y en el proceso de la enfermedad, entre los que se encuentran edad avanzada, la hipertensión arterial, el colesterol elevado, obesidad y sedentarismo, diabetes, entre otros; así como los factores cognitivos, emocionales y conductuales, en este caso la depresión y el manejo del estrés (GeoSalud, 2000). Estos últimos se asocian en la medida en que las evaluaciones que la persona realice de las situaciones, a 
nivel del pensamiento influirán tanto en el estado de ánimo como en la conducta (Beck, 2000). Esto cobra relevancia ya que durante la enfermedad las personas que tienen dificultades para el manejo del estrés, volviéndose crónico, presentan también síntomas depresivos (Sandín, 1993).

Considerando lo antes dicho, este estudio contrastar las siguientes hipótesis:

\section{Hipótesis General}

H1: Existe una relación estadísticamente significativa entre la depresión y los estilos de afrontamiento al estrés en un grupo de pacientes con enfermedad cerebrovascular.

\section{Hipótesis Específicas}

H1: Existe una relación estadísticamente significativa entre la depresión y los estilos de afrontamiento al estrés enfocados en el problema, en un grupo de pacientes con enfermedad cerebrovascular.

H2: Existe una relación estadísticamente significativa entre la depresión y los estilos de afrontamiento al estrés enfocados en la emoción, en un grupo de pacientes con enfermedad cerebrovascular.

H3: Existe una relación estadísticamente significativa entre la depresión y los estilos de afrontamiento al estrés evitativos, en un grupo de pacientes con enfermedad cerebrovascular.

\section{MÉTODO}

\section{Diseño de investigación}

Se empleó el método descriptivo y fue de tipo correlacional, de acuerdo con la clasificación de Sánchez y Reyes (2006). El diseño de investigación que se empleó fue correlacional, el cual permitió establecer el grado de relación existente entre la depresión y los estilos de afrontamiento al estrés.

\section{Población y muestra}

La población estuvo conformada por el total de pacientes atendidos en el servicio de neurología del hospital nacional Daniel A. Carrión, diagnosticados con enfermedad cerebrovascular.

La muestra estuvo comprendida por 50 personas de ambos sexos -35 hombres y 15 mujeres-, diagnosticadas con enfermedad cerebrovascular, 25 diagnosticadas con hemorragia y las otras 25 con isquemia. Las edades se distribuyeron entre los 45 y 90 años, siendo el rango entre los 61 y 75 el de mayor porcentaje (46\%).

La muestra de estudio fue seleccionada; según su representatividad, bajo el criterio de la investigadora el cual se ajustó a los fines del estudio. 
Los criterios de inclusión para la muestra de estudio fueron:

1. Presentar enfermedad cerebrovascular.

2. Ser atendido en consulta externa.

3. Edad comprendida entre 40 y 90 años.

4. De ambos sexos

5. Que sus condiciones mentales no constituyan impedimento para la comprensión y aplicación de los inventarios.

Las variables del estudio fueron: estilos de afrontamiento al estrés y depresión.

\section{Instrumentos}

Entrevista semiestructurada, la cual recogió información de aspectos demográficos como: sexo, edad, grado de instrucción, religión, y diagnóstico o tipo de enfermedad cerebrovascular, este último se corroboraba con la revisión de la historia clínica.

\section{El Inventario de Estimación de Afrontamiento COPE Breve en su forma} disposicional de Carver (1997), consta de 28 ítems tipo likert que oscila entre 1 (no en absoluto) hasta 4 (suelo hacer esto mucho). Tiene como objetivo evaluar las respuestas habituales de las personas ante situacionesl estresantes; presenta 3 estilos de afrontamiento.

En la presente investigación, se evaluó la validez mediante el criterio de jueces, obteniéndose el $100 \%$ de ítemes válidos; en cuanto a la confiabilidad, en los estilos de afrontamiento centrado en el problema, en la emoción y los evitativos se obtuvo un alfa de Cronbach $0.66,0.46$ y 0.51 y respectivamente. La confiabilidad referente al Estilo de Afrontamiento Centrado en el Problema obtiene un valor Alfa de Cronbach de .66 indicando así mayor consistencia, seguido por los estilos de Afrontamiento Evitativos con un valor alfa de .51, el valor que se encuentra por debajo de lo esperado se observa en los Estilos de Afrontamiento Centrados en la Emoción con un valor de .46.

Inventario de Depresión de Beck (IDB) elaborado por Beck en 1961 y adaptado en el Perú por Novara, Sotillo y Wharton (1985). El instrumento consta de 21 ítems para evaluar la intensidad sintomática y las manifestaciones conductuales de la depresión, se compone de 4 afirmaciones, desde 0 ausente o mínima, hasta 3 muy intenso o grave.

En el Perú ha sido adaptado por Novara, Sotillo y Wharton (1985), quienes utilizaron el método de validez relacionada con el criterio obteniéndose un coeficiente de correlación de Pearson, de 0.74 respecto a la evaluación hecha por el psiquiatra, así mismo se obtuvo un valor de 0.76 con relación a la escala de depresión de Zung y una correlación de 0.72 con la escala de depresión de Hamilton.

En la presente investigación, se evaluó la validez de contenido mediante el criterio de jueces, obteniéndose el $100 \%$ de ítemes válidos; así mismo, se obtuvo un 
coeficiente de confiabilidad alfa de Cronbach de .89. Respecto a la confiabilidad, se observa que todos los valores de la correlación ítem-total corregida, obtienen coeficientes mayores a .20 y un Alfa de Cronbach por encima de .70 (Kline, 1993).

\section{RESULTADOS}

\section{Resultados descriptivos}

Los resultados muestran que, en cuanto al estilo de afrontamiento centrado en el problema, 33 personas manifestaron hacer uso generalmente de estos, se observa además que 12 personas indicaron utilizar estos estilos de afrontamiento siempre, como se muestra en la Tabla $\mathrm{N}^{\circ} 1$.

Tabla 1. Distribución según estilos de afrontamiento centrado en el problema

\begin{tabular}{lcc}
\hline & Frecuencia & $\%$ \\
\hline Nunca & 1 & 2 \\
A veces & 4 & 8 \\
Generalmente & 33 & 66 \\
Siempre & 12 & 24 \\
Total & 50 & 100 \\
\hline
\end{tabular}

Respecto al estilo de afrontamiento centrado en la emoción la Tabla N. ${ }^{\circ} 2$ muestra que, 34 personas de la muestra indican que hacen uso de este estilo generalmente, sin embargo, otras 15 mencionan hacerlo solo a veces.

Tabla 2. Distribución según estilos de afrontamiento centrado en la emoción

\begin{tabular}{lcc}
\hline & Frecuencia & $\%$ \\
\hline Nunca & 0 & 0 \\
A veces & 15 & 30 \\
Generalmente & 34 & 68 \\
Siempre & 1 & 2 \\
Total & 50 & 100 \\
\hline
\end{tabular}

Sin embargo, se observa en la Tabla $\mathrm{N}^{\circ} 3$ que respecto a la negación como estilo de afrontamiento centrado en la emoción, 21 de los participantes informan que nunca la utilizan, mientras que 22 de ellos, solo a veces afrontan negando los hechos.

Tabla 3. Distribución según la negación como estilo de afrontamiento centrado en la emoción

\begin{tabular}{lcc}
\hline & Frecuencia & $\%$ \\
\hline Nunca & 21 & 42 \\
A veces & 22 & 44 \\
Generalmente & 7 & 14 \\
Siempre & 0 & 0 \\
Total & 50 & 100 \\
\hline
\end{tabular}


Por otro lado, se puede apreciar en la Tabla N. ${ }^{\circ} 4$ que el estilo de afrontamiento evitativo es el menos utilizado, ya que 40 de las personas que representan el $80 \%$ de la muestra, indican que sólo a veces hacen uso de estos, mientras que 8 de ellos los utilizan generalmente y dos personas mencionan que nunca han realizado las estrategias de afrontamiento representativas de este grupo de estilos.

Tabla 4. Distribución según estilos de afrontamiento evitativos

\begin{tabular}{lcc}
\hline & Frecuencia & $\%$ \\
\hline Nunca & 2 & 4 \\
A veces & 40 & 80 \\
Generalmente & 8 & 16 \\
Siempre & 0 & 0 \\
Total & 50 & 100 \\
\hline
\end{tabular}

En la Tabla N. ${ }^{\circ} 5$, se presenta el estilo de afrontamiento evitativo específico, enfocar y liberar emociones, en el que la muestra indica que es utilizado generalmente por 22 de ellos, mientras que 23 participantes reportan que la utilizan siempre.

Tabla 5. Distribución según estilo evitativo, enfocar y liberar emociones

\begin{tabular}{lcc}
\hline & Frecuencia & $\%$ \\
\hline Nunca & 2 & 4 \\
A veces & 3 & 6 \\
Generalmente & 22 & 44 \\
Siempre & 23 & 46 \\
Total & 50 & 100 \\
\hline
\end{tabular}

Finalmente, en la Tabla N ${ }^{\circ} 6$ se presenta el estilo de afrontamiento evitativo, uso de sustancias, el cual es referido por 45 de los participantes que representan el $90 \%$, que nunca es utilizado; mientras que 4 de ellos mencionan hacerlo a veces y sólo uno a veces.

Tabla 6. Distribución según estilo evitativo, uso de sustancias

\begin{tabular}{lcc}
\hline & Frecuencia & $\%$ \\
\hline Nunca & 45 & 90 \\
A veces & 4 & 8 \\
Generalmente & 1 & 2 \\
Siempre & 0 & 0 \\
Total & 50 & 100 \\
\hline
\end{tabular}

\section{Resultados inferenciales}

El contraste de la primera hipótesis que indica que existe una relación estadísticamente significativa entre la depresión y los estilos de afrontamiento al estrés 
enfocados en el problema, en un grupo de pacientes con enfermedad cerebrovascular (Tabla $\mathrm{N}^{\circ} 7$ ), muestra como resultado que no existen correlaciones altamente significativas, entre las variables mencionadas.

Es así que presenta una dirección negativa, un valor de fuerza muy bajo y no muestra diferencias estadísticamente significativas $(\mathrm{r}=-0.06, \mathrm{p}<.01)$.

Respecto a la segunda hipótesis, la cual indica que existe una relación estadísticamente significativa entre la depresión y los estilos de afrontamiento al estrés enfocados en la emoción, en un grupo de pacientes con enfermedad cerebrovascular del servicio de neurología del hospital Daniel Alcides Carrión del Callao. Obtuvo como resultado, mediante el contraste de hipótesis, que no existen correlaciones altamente significativas, entre las variables mencionadas.

De este modo, entre las variables depresión y estilo de afrontamiento al estrés centrado en la emoción, se obtuvo una dirección negativa, un valor de fuerza muy bajo y no muestra diferencias estadísticamente significativas $(\mathrm{r}=-0.16, \mathrm{p}<.01)$.

Finalmente, la tercera hipótesis, la cual indica que existe una relación estadísticamente significativa entre la depresión y los estilos de afrontamiento al estrés evitativos, en un grupo de pacientes con enfermedad cerebrovascular del servicio de neurología del Hospital Daniel Alcides Carrión del Callao, dio como resultado que sí existen correlaciones significativas entre las variables antes mencionadas.

Así se tiene que las variables depresión y los estilos de afrontamiento al estrés evitativos muestran una correlación positiva, fuerza media y diferencia estadísticamente significativas $(\mathrm{r}=0.54, \mathrm{p}<.01)$.

Tabla 7. Correlaciones rho de Spearman entre las variables depresión y estilos de afrontamiento al estrés en pacientes con enfermedad cerebrovascular

\begin{tabular}{lccc}
\hline & $\begin{array}{c}\text { Afrontamiento Centrado } \\
\text { en el Problema }\end{array}$ & $\begin{array}{c}\text { Afrontamiento Centrado } \\
\text { en la Emoción }\end{array}$ & $\begin{array}{c}\text { Afrontamiento } \\
\text { Evitativo }\end{array}$ \\
\hline Depresión & -0.06 & -0.16 & $0.54^{* *}$ \\
\hline$* * \mathrm{p}<.01$ & & & \\
$\mathrm{n}=50$ & & &
\end{tabular}

\section{DISCUSIÓN}

El análisis de la hipótesis H1 indica que esta no se valida ya que no existe relación estadísticamente significativa entre la depresión y los estilos de afrontamiento al estrés enfocados en el problema en los pacientes con enfermedad cerebrovascular, al respecto los resultados indican que las personas de la muestra utilizan con alta frecuencia los estilos de afrontamiento centrados en el problema (Tabla N. ${ }^{\circ}$ ), ello alude a que la mayor parte de participantes en el estudio, regularmente afrontan la realidad esforzándose para reducir la fuente del estrés, es decir, utilizan un afrontamiento activo, planifican la mejor manera de manejar los problemas y realizan 
las acciones pertinentes para lograr este objetivo, de igual modo buscarán apoyo social, es decir, consejo, asistencia e información; en consecuencia, son capaces de enfocar su atención concentración y demás capacidades en respuestas centradas a resolver el problema. Así, las personas no deprimidas pueden tener pensamientos negativos, sin embargo, estas no pierden la capacidad de afrontar, analizar y rechazar esos pensamientos negativos como lo menciona, Buendía (1993). Desde la perspectiva de estos aportes, se presenta una correlación negativa (Tabla $\mathrm{N}^{\circ} 7$ ) entre las variables estilos de afrontamiento centrado en el problema y la depresión, pero que no llega a ser significativa, por tanto resultaría importante realizar otros estudios que permitan deslindar y clarificar relaciones entre las variables depresión y estilos de afrontamiento centrados en el problema.

Respecto al análisis de la hipótesis $\mathrm{H} 2$, indica que esta no se valida puesto que no existe relación estadísticamente significativa entre la depresión y los estilos de afrontamiento al estrés enfocados en la emoción, en un grupo de pacientes con enfermedad cerebrovascular del servicio de neurología del hospital nacional Daniel Alcides Carrión del Callao.

El que la muestra estudiada también utilice con frecuencia estas estrategias de afrontamiento (Tabla $\mathrm{N}^{\circ} 2$ ), podría contribuir en mejoras para su salud, ya que además la estrategia que consiste en negar la existencia del problema fue utilizada con menor frecuencia como se muestra en la Tabla $\mathrm{N}^{\circ} 3$. Es así que, FernándezConcepción y cols. (2001) encontraron que la aceptación de los problemas y la reinterpretación positiva de estas, presentaron una relación positiva con la energía y la movilidad física, es decir que estas estrategias utilizadas por los pacientes con ECV facilitarían un mejor nivel de desenvolvimiento y/o funcionamiento, más aún si presentan alguna secuela física. Estos autores también mencionan que un mayor apoyo social percibido, especialmente en el área de la salud fomenta que las personas presenten mejor calidad de vida en aspectos como el dolor, sueño y aislamiento social.

Otra de las estrategias de afronte centrados en la emoción relevantes que se podrían citar, es enfocarse en la religión, estas estrategias utilizadas generalmente en los participantes del presente estudio podrían favorecer una reevaluación de la situación que conlleve a una mejor adaptación ante la enfermedad, pues como Koening, George \& Siegler (citados en Rivera y Montero, 2007) indican el acudir a la religión juega un papel importante en la conservación de la salud en adultos mayores, al relacionarse con el sentido de bienestar personal. Aunque los resultados muestran una correlación negativa esta no es significativa por lo que un estudio posterior podría contribuir a esclarecer la información.

Con relación al análisis de la hipótesis $\mathrm{H} 3$ indica que esta es válida, pues las variables, depresión y los estilos de afrontamiento al estrés evitativos, en un grupo de pacientes con enfermedad cerebrovascular del servicio de neurología del hospital nacional Daniel Alcides Carrión del Callao, se correlacionan de manera significativa y positiva $(\mathrm{r}=0.54)$. Estos resultados evidencian que los estilos de 
afrontamiento evitativos son interdependientes de la depresión, desde este aspecto la persona con enfermedad cerebrovascular que muestra mayor intensidad de síntomas depresivos, utiliza un estilo de afrontamiento que incluye estrategias evitativas específicas como el liberar emociones centrándose en los aspectos desagradables, disminuir los esfuerzos o abandonar sus metas para manejar el estresor, distrayendo su actividad hacia otros objetivos, culparse a sí mismo por lo sucedido o utilizando sustancias psicoactivas.

Como menciona Beck (1983), la valoración que se preste a un acontecimiento es un elemento clave en la depresión, en la presente investigación se resalta también que influye en el estilo de afrontamiento ante el estrés que la persona utilice.

Cabe señalar que la mayoría de las personas que participaron en el presente estudio, utilizan solo, a veces, los estilos de afrontamiento evitativos (Tabla $\mathrm{N}^{\circ} 4$ ). Analizando estos aspectos relacionándolos con las estrategias propias del estilo de afrontamiento evitativo, se observa que respecto a los contenidos relativos a la visión que tiene sobre sí mismo, la persona que se autovalora negativamente, se percibe menos capacitada que los demás, esta distorsión de la realidad hace factible explicarse por qué los pacientes con enfermedad cerebrovascular de la presente investigación, centrándose en una visión negativa de sí, no suelen afrontar o evitan los sucesos evaluados como amenazantes, tendiéndose a hacer reproches injustificados, en relación a lo mencionado por Beck (1983), las distorsiones de pensamiento que son desadaptativas en las personas depresivas se manifiesta en la tendencia a responsabilizarse de los resultados negativos, autoculparse, y por el contrario se niegan a asumir sus logros. Si añadido a ello tiene una visión de su ambiente así como de sus propias experiencias, pensando que no merece ser apreciado o querido, además se considera impotente ante las dificultades aunque estas sean pequeñas, y si finalmente su visión de futuro y del mundo, es negativa y sin esperanza; entonces la persona tenderá a evitar las consecuencias desagradables que le traerá el afrontar el suceso, por lo que opta -bajo las características antes mencionadas- por un desentendimiento mental y conductual de los eventos adversos, distrayendo sus actividades hacia otros objetivos que no son relevantes para manejar la situación, centrándose solo en las consecuencias emocionales desagradables descargándolas, disminuye o incluso abandona sus esfuerzos, ya que piensa que cualquier esfuerzo que realice será en vano, pues no hay salida, estas dos últimas, enfocar y liberar emociones y el desentendimiento conductual son consideradas las estrategias más desadaptativas por Giles, Llull, y Richard's, (2001). Al respecto, el análisis del tipo de afrontamiento enfocar y liberar emociones, evidencia que es utilizada generalmente y siempre dentro de la muestra de estudio (Tabla N. ${ }^{\circ}$ ), lo que indica que los pacientes, que muestran síntomas depresivos tienden a enfocarse en descargar emociones negativas, lo que podría ser perjudicial, pues ello no les llevaría a mejorar su situación.

Cabe hacer mención que, dentro de los estilos evitativos, el menos utilizado (Tabla $\mathrm{N} .{ }^{\circ} 6$ ) por los pacientes con ECV es el uso de sustancias psicoactivas, probablemente por su propia condición de salud. 
Como se ha explicado ya, el afrontamiento evitativo está relacionado con la depresión, es decir, la presencia de síntomas depresivos o por el contrario, la ausencia de estos influirán en la manera cómo se afronte la ECV, aportes como el de Rojas (2002), muestran la influencia de la depresión en detrimento de la recuperación física, en pacientes con secuela motora por enfermedad cerebrovascular. Es así que el estado de salud del organismo dependerá mucho de la interacción cogniciónemoción-afrontamiento (Buendía, 1993; Sandín, 1993).

Lo mismo ocurre en sentido inverso al darse una interdependencia entre afrontamiento y depresión, pues como indica Buendía (1993), cuando se mantienen estilos de afrontamiento desadaptativos, se presentan diversas alteraciones psicológicas, como es el caso de la depresión. Estas características que, aunque solo se presentan a veces, según evidencian los resultados complementarios de esta investigación, alertan acerca de las precauciones y acciones que son importantes tomar al respecto en los pacientes con enfermedad cerebrovascular, pues pueden mellar los avances en una rehabilitación o a la adherencia al tratamiento, al impedir a la persona, el utilizar estrategias adaptativas en favor de su salud.

\section{CONCLUSIONES}

1. La hipótesis H1 no se valida en la presente investigación, puesto que no existen relaciones estadísticamente significativas entre las variables, depresión y estilos de afrontamiento al estrés enfocados en el problema, en un grupo de pacientes con enfermedad cerebrovascular del servicio de neurología del hospital nacional Daniel Alcides Carrión del Callao.

2. La hipótesis $\mathrm{H} 2$ no se valida en el presente estudio, pues no existen relaciones estadísticamente significativas entre las variables, depresión y estilos de afrontamiento al estrés enfocados en la emoción, en un grupo de pacientes con enfermedad cerebrovascular del servicio de neurología del hospital nacional Daniel Alcides Carrión del Callao.

3. La hipótesis $\mathrm{H} 3$ es válida, ya que existen relaciones estadísticamente significativas y positivas, entre las variables, depresión y estilos de afrontamiento al estrés evitativos, en un grupo de pacientes con enfermedad cerebrovascular del servicio de neurología del Hospital Nacional Daniel Alcides Carrión del Callao.

\section{REFERENCIAS BIBLIOGRÁFICAS}

Arrazola, A., Beguiristain, J. M., Garitano, B., Mar, J., y Elizalde, B. (2005). Atención hospitalaria a la enfermedad cerebrovascular aguda y situación de los pacientes a los 12 meses. Rev Neourol, 40, 326-330. Extraído el 8 de mayo del 2007 desde http:// www.neurologia.com/pdf/Web/4006/s060326.pdf

Área de Procesamiento de Datos del Hospital Nacional Daniel Alcides Carrión (2009). Base de Datos. Lima: HNDAC.

Beck, A. (1983). Terapia cognitiva de la depresión. Bilbao: Desclee de Brouwer. 
Beck, J. (2000). Terapia cognitiva: Conceptos básicos y profundización. Barcelona: Gedisa.

Buendía, J. (1993). Estrés y depresión. En J. Buendía (coord.), Estrés y psicopatología (pp. 97-111). Madrid: Ediciones Pirámide.

Carver, C. S., Sheier, M. F., \& Weintraub, J. K. (1989). Assesing Coping strategies: A theoretically based approach. Journal of Personality an Social Psychology, 56, 267-283.

Carver, C. S. (1997). You want to measure coping but your protocols too long: Consider The Brief COPE. International Journal of Behavioral Medicine, 4, 92-100.

Fernández-Concepción, O., Fiallo-Sánchez, M. C., Álvarez-González, M. A., Roca, M. A., Concepción-Rojas, M., y Chávez, L. (2001). La calidad de vida del paciente con accidente cerebrovascular: Una visión desde sus posibles factores determinantes. Rev Neurol, 32, 725-731. Obtenido el 11 de agosto del 2010 desde http://www.neurologia. com/pdf/Web/3208/k080725.pdf.

GeoSalud (2000). Accidente cerebrovascular. Extraído el 4 de agosto del 2007 desde http://www.geosalud.com/Enfermedades \% 20Cardiovasculares/accidente \% 20 cerebrovascular.htm

Giles, M. V., Llull, D., y Richard's, M. (2001). Estrategias de afrontamiento y situaciones estresoras en pacientes internados por enfermedades cardiovasculares. Extraído el 5 de julio del 2007 desde www.sames.org.ar/web/art_news_vol 6-02. htm

Lazarus, R., y Folkman, S. (1986). Estrés y Procesos Cognitivos. Barcelona: Martínez Roca.

Novara, J., Sotillo, C., y Wharton, D. (1985). Estandarización de las Escalas de Beck, Hamilton y Zung para depresión. Lima: Instituto de Salud Mental Honorio DelgadoHideyo -Noguchi.

Organización Mundial de la Salud. (1992). Décima Revisión de la Clasificación Internacional de las Enfermedades. Trastornos Mentales y del Comportamiento. Madrid: Técnicas Gráficas Forma.

Pereyra, M. (2006). Estrés y Salud. En Oblitas, L. A., Psicología de la salud y calidad de vida (pp. 213-248). International Thomson Editores.

Rivera, A. y Montero, M. (2007). Medidas de afrontamiento religioso y espiritualidad en adultos mayores mexicanos. Salud Mental, 30, 39-47. Extraído el 18 noviembre del 2010 desde http://redalyc.uaemex.mx/pdf/582/58230106.pdf

Rojas, E. (2002). Influencia de la depresión en la recuperación física de los pacientes con secuela motora de enfermedad vascular cerebral. Tesis para optar el grado de Magíster en Medicina, Universidad Nacional Mayor de San Marcos. Lima.

Sánchez, H., y Reyes, C. (2006). Metodología y diseños en la investigación científica. Lima: Visión Universitaria.

Sandín, B. (1993). Estrés y salud: Factores que intervienen en la relación entre el estrés y la enfermedad física. En J. Buendía (coord.), Estrés y psicopatología (pp. 148-175). Madrid: Ediciones Pirámide. 
Surtees, P. G., Wainwright, N. W. J., Luben, R. L., Wareham, N. J., Bingham, S. A. \& Khaw, K. T. (2007). Adaptation to social adversity is associated with stroke incidente: Evidence from the EPIC-Norfolk prospective cohort study. Stroke, 38, 1447-1453. Extraído el 11 de junio del 2008 desde http://pt.wkhealth.com/pt/re/ stroke/pdfhandler.00007670-200705000-00016. pdf;jsessionid=N8fQPJndLyjL8ry gcZYvprv4tPxkdw2H6yyXFFJ7FktpdQjKGqgG!1507367712!181195629!8091!-1

Surtees, P. G., Wainwright, N. W. J., Luben, R. L., Wareham, N. J., Bingham, S. A. \& Khaw, K. T. (2008). Psychological distress, major depressive disorder, and risk of stroke. Neurology, 70, 788-794.

Velásquez A. (2006). Análisis del estudio de carga de enfermedad en el perú-2004 y propuesta metodológica para el ajuste con datos nacionales de morbilidad. MINSA Perú: Promoviendo alianzas y estrategias, Abt Associates Inc. Pp. 64. 